\title{
Choosing wisely: a model-based analysis evaluating the trade-offs in cancer benefit and diagnostic referrals among alternative HPV testing strategies in Norway
}

\author{
Emily A Burger ${ }^{\star}, 1,3$, Kine Pedersen ${ }^{2,3}$, Stephen Sy ${ }^{1}$, Ivar Sønbø Kristiansen ${ }^{2}$ and Jane J Kim ${ }^{1}$ \\ ${ }^{1}$ Harvard T.H. Chan School of Public Health, Center for Health Decision Science, 718 Huntington Avenue, 2nd Floor, Boston, MA \\ 02115, USA and ${ }^{2}$ University of Oslo, Department of Health Management and Health Economics, PO BOX 1089 Blindern, Oslo \\ 0317, Norway
}

Background: Forthcoming cervical cancer screening strategies involving human papillomavirus (HPV) testing for women not vaccinated against HPV infections may increase colposcopy referral rates. We quantified health and resource trade-offs associated with alternative HPV-based algorithms to inform decision-makers when choosing between candidate algorithms.

Methods: We used a mathematical simulation model of HPV-induced cervical carcinogenesis in Norway. We compared the current cytology-based strategy to alternative strategies that varied by the switching age to primary HPV testing (ages 25-34 years), the routine screening frequency (every 3-10 years), and management of HPV-positive, cytology-negative women. Model outcomes included reductions in lifetime cervical cancer risk, relative colposcopy rates, and colposcopy rates per cervical cancer prevented.

Results: The age of switching to primary HPV testing and the screening frequency had the largest impacts on cancer risk reductions, which ranged from $90.9 \%$ to $96.3 \%$ compared to no screening. In contrast, increasing the follow-up intensity of HPVpositive, cytology-negative women provided only minor improvements in cancer benefits, but generally required considerably higher rates of colposcopy referrals compared to current levels, resulting in less efficient cervical cancer prevention.

Conclusions: We found that in order to maximise cancer benefits HPV-based screening among unvaccinated women should not be delayed: rather, policy makers should utilise the triage mechanism to control colposcopy referrals.

Similar to many countries around the world, cervical cancer prevention strategies in Norway involve both primary and secondary measures. In 2009, Norway introduced the quadrivalent human papillomavirus (HPV) vaccination, offered to all 12-yearold girls, free of charge; however, screening will continue to remain an essential preventive approach, particularly among unvaccinated women. New screening technologies that involve switching from cytology to primary HPV DNA testing are actively being considered by several countries, including Norway. There is concern, however, that HPV testing may increase the demand for colposcopy resources (e.g., colposcopists and pathologists) due to both the enhanced sensitivity and reduced specificity in detecting high-grade lesions. A strategy to reduce excess referral involves restricting the age at which women begin primary HPV

\footnotetext{
*Correspondence: Dr EA Burger; E-mail: eburger@hsph.harvard.edu

${ }^{3}$ These authors contributed equally to this work.
}

Received 9 February 2017; revised 16 May 2017; accepted 6 July 2017; published online 3 August 2017

(C) 2017 Cancer Research UK. All rights reserved 0007-0920/17 
testing, as transient HPV infections occur more often in younger women. For example, a randomised implementation pilot study in Norway is currently evaluating a screening strategy that involves waiting until age 34 years to switch from cytology to HPV testing, which may increase the discriminatory power and place less strain on limited resources. However, analyses have shown that an earlier switch age (i.e. 30 or 31 years) may be more effective (Ronco et al, 2014) and cost-effective (Burger et al, 2012) compared to switching at age 34 or 35 years. Alternative approaches to control colposcopy referrals involve identifying the optimal management of HPVpositive women either using HPV genotyping or cytology triage (Cuzick et al, 2006). In Norway, the initial primary HPV screening proposal (Cancer Registry of Norway, 2011) required multiple persistent HPV-positive, cytology-negative results prior to prompting colposcopy referral, while the current implementation study protocol (Nygard et al, 2013) only requires a single persistent result to prompt colposcopy referral. Appropriate management of screenpositive women is important not only to ensure efficient use of resources, but to also limit anxiety related to positive test results and diagnostic work-up, as well as the potential adverse events associated with treatment of precancerous lesions (e.g., preterm delivery and low birth weight (Jin et al, 2014)).

When considering new technologies, financial considerations and formal cost-effectiveness analyses help inform one aspect of the decision-making process; however, other factors such as quantifying resource use and identifying capacity constraints play an important role in establishing the feasibility of population-based implementation. While other modelling studies have quantified resource use (Stout et al, 2008; Naber et al, 2016; Smith et al, 2016), to our knowledge, isolating the impact primary HPV-based screening and alternative triage approaches have on colposcopy referrals and health benefits have not been explored previously. Our objective was to enumerate the trade-offs in health benefits and resource use associated with adopting primary HPV testing strategies for unvaccinated women using a decision-analytic model. In this context we explored how alternative ages to switch to primary HPV testing, routine screening frequency (i.e., interval length) and the follow-up intensity of women HPV-positive with no cytologic abnormalities affects resource use, referrals, and health benefits in relation to current levels in Norway.

\section{METHODS}

Analytic approach. We used a previously developed mathematical simulation model of HPV-induced cervical carcinogenesis (Kim et al, 2007; Campos et al, 2014; Burger et al, 2016; Pedersen et al, 2016) to evaluate the trade-offs in long-term health benefits and colposcopy referral rates associated with implementing primary HPV testing in Norway. We compared the current Norwegian triennial cytology-based screening programme (starting at age 25 years) with candidate strategies that involve switching from cytology to HPV DNA testing. We varied the age of switching from cytology to primary HPV testing (i.e., at age $25,28,31$, or 34 years), the primary screening frequency of HPV testing (i.e. ranging from 3 to 10 years), and considered alternative approaches for management of HPV-positive, cytology-negative (HPV + I Cyt - ) women. Model outcomes included the lifetime risk of developing cervical cancer, the total number of women who developed cervical cancer, the total number of cytology and HPV tests, the total number of precancer treatments, and the total number of colposcopy referrals. Outcomes are reported over the lifetime of each cohort and may reflect single or multiple interactions, e.g., an individual woman may experience multiple colposcopies over her lifetime while under surveillance. For each strategy, we estimated the reduction in lifetime risk of developing cervical cancer compared to (i) current guidelines and (ii) no screening, the relative colposcopy referral rates compared with the current Norwegian guidelines, and the additional number of colposcopy referrals per additional number of cervical cancer case prevented, compared to (i) current guidelines and (ii) no screening.

Decision-analytic model. The decision-analytic model (Kim et al, 2007; Campos et al, 2014; Burger et al, 2016; Pedersen et al, 2016) simulates a hypothetical cohort of individual healthy girls over their lifetime, starting at age nine years, who at each month, face probabilities of type-specific HPV incidence and clearance, progression and regression of precancerous lesions, and progression to cervical cancer. Transition probabilities can be a function of duration of infection or lesion, age, and HPV genotype. The model is stratified by health states distinguished by HPV type (i.e., healthy, HPV-16, -18, -31, -33, -45, -52 and -58 , other pooled highrisk types, and pooled low-risk types), cervical intraepithelial neoplasia (CIN) grades 2 and 3 (CIN2/3), and invasive cancer by stage (i.e., local, regional, distant). Women with cancer face stagespecific survival rates, while all women face Norway-specific competing mortality risks from other causes (Statistics Norway, 2015). For each unique screening strategy, the model tracks and records the disease and screening history for each individual woman, such as the number of colposcopy referrals, treated CIN2/ 3 lesions, and detected cervical cancers.

Details of the model structure, calibration and validation to the Norwegian context have been described elsewhere (Burger et al, 2012, 2016; Pedersen et al, 2016; Norwegian Technical Appendix, 2017). Briefly, we calibrated the model to empirical data using a likelihood-based scoring algorithm to identify parameter values that maximised correspondence between model outputs and epidemiologic data from Norway. Norwegian-specific calibration targets included age- and type-specific HPV prevalence, and HPV type-distribution within precancerous lesions and cancer. Under current patterns of screening behaviour in Norway, the model projected outcomes that correspond to observed Norwegian data not used to inform the model, including age-specific cervical cancer incidence (Norwegian Technical Appendix, 2017) and colposcopy referral rates (Supplementary Figure 1). To capture parameter uncertainty, all analyses were conducted with 50 goodfitting parameter sets; summary statistics were reported as the average outcome across the 50 parameter sets, and uncertainty bounds (UB) reflecting the minimum and maximum value.

Screening algorithms. We compared the current Norwegian cytology-based screening programme to candidate strategies that involve primary HPV testing (Burger et al, 2012; Nygard et al, 2013). The current Norwegian strategy invites all women aged 25 to 69 years to triennial cytology-based screening. Women with cytology results suggestive of minor cervical lesions (i.e. atypical squamous cells of undetermined significance (ASC-US) or lowgrade squamous intraepithelial lesion (LSIL)) and positive for a high-risk HPV infection on reflex testing are triaged using repeat co-testing (i.e., cytology and HPV testing) 6-12 months later, while women with high-grade cytologic results (high-grade intraepithelial neoplasia (HSIL)) are referred directly to colposcopy with biopsy, and treatment if the biopsy indicates CIN2 or worse. For this analysis we assumed excisional treatment was successful and prompted increased surveillance prior to returning women to routine screening. The proposed HPV-based algorithm in Norway involves switching women from the current cytology-based strategy at age $\geqslant 34$ years to primary HPV DNA testing. Women who are HPV-negative can return to routine screening at 5-yearly intervals, while HPV-positive women are re-tested using liquidbased cytology (LBC). HPV-positive women with a cytology result of ASCUS or more severe are referred directly to colposcopy with biopsy, while women with a normal cytology result receive a new HPV test in 12 months. At the 12-month visit, HPV-positive 
women are referred directly to colposcopy with biopsy, while HPVnegative women can return to routine screening.

We evaluated $216 \mathrm{HPV}$-based screening strategies relevant across multiple settings, including the algorithm currently under evaluation in Norway. We compared variations in the HPV-based strategy algorithm that differed by the primary screening frequency $(3,4,5,6,8$ or 10 years) across four alternative ages that women switch from cytology- to HPV-based screening (ages 25, 28, 31 or 34 years) (Figure 1). We assumed that women testing HPVnegative return to the routine screening at the specified primary screening frequency, while women testing positive for a high-risk HPV infection are re-tested using LBC. HPV-positive women with a cytology result of ASCUS or more severe are referred directly to colposcopy with biopsy. $\mathrm{HPV}+/ \mathrm{Cyt}-$ women would receive a new HPV test at 12 months, followed by a reflex cytology for HPVpositive women. As uncertainty remains regarding the appropriate management of HPV + /Cyt - women, we evaluated alternative management strategies for this sub-group of women, including scenarios that varied by the number of persistent HPV + /Cyt results required to prompt referral to colposcopy $(1,2$ or 3 persistent HPV + /Cyt - result(s)), and wait time between repeat testing $(6,12$ or 18 months) (Figure 1). We assumed that an HPVnegative result prompts return to routine screening; and if at any point, a woman is both HPV-positive and cytology-positive ( $\geqslant$ ASCUS), she is immediately referred to colposcopy with biopsy. For all strategies, screening was initiated at age 25 years and ended at age 71 to allow a consistent pattern of screening end ages across the variations in screening scenarios. Our analysis assumed $100 \%$ screening coverage and follow-up in order to assess the maximum differences among strategies and to avoid imposing assumptions regarding the distribution of screening coverage for extended screening intervals, for which there are no empirical data. As the baseline comparator strategy will influence relative colposcopy rates, we repeated calculations using cytology-based screening currently recommended by the United States Preventative Services Task Force in the United States (US) (Moyer, 2012) in uncertainty analysis. This strategy involved referral of women with LSIL or more severe results directly to colposcopy with biopsy.

The model values and plausible ranges for LBC and HPV test characteristics were based on published literature and varied in sensitivity analysis (Nanda et al, 2000; Mayrand et al, 2007; Arbyn et al, 2008; Ronco et al, 2014). For LBC, we assumed a sensitivity of $70 \%$ to detect CIN2 or more severe, and a specificity of $91 \%$ (Nanda et al, 2000; Mayrand et al, 2007; Arbyn et al, 2008). The sensitivity (specificity) of HPV DNA assays, defined as the probability of HPV DNA-positive (-negative) given HPV DNA is present (absent), is $100 \%$, but we varied this assumption in uncertainty analysis. The resulting clinically relevant sensitivity of high-risk HPV testing to detect CIN2 or worse, which is a model validation output, was 93\% (UB: $88-97 \%$ ), and the clinically relevant specificity was $80 \% \quad(78.7-83.1 \%)$, consistent with empirical studies (Mayrand et al, 2007). In univariate uncertainty analysis, we reduced the clinical HPV test sensitivity to $84 \%$. The diagnostic accuracy of colposcopy with biopsy was based on data from the New Mexico HPV Pap Registry (Stoler et al, 2015b); we evaluated the impact of perfect sensitivity and specificity in univariate uncertainty analysis.

\section{RESULTS}

Primary analysis. Reductions in lifetime cervical cancer risk associated with the alternative HPV-based strategies ranged from $90.9 \%(90.0-92.1 \%)$ to $96.3 \%(95.8-96.8 \%)$ compared to no screening, and provided greater reductions than the current Norwegian cytology-based approach (i.e., $87.7 \%$ (86.6-88.7\%)) (Figure 2, Supplementary Figures 2 and 3). The age of switching to primary HPV testing had the largest impact on cancer risk reductions, while the primary screening frequency had a modest influence on cancer risk reductions. For example, delaying HPVbased screening initiation from age 25 years to age 34 years yielded 2.6-3.3\% absolute decrease in the risk reductions, while lengthening the primary screening frequency from 3 to 10 years was associated with a $0.9-2.4 \%$ absolute decrease in the risk reductions. In contrast, increasing the follow-up intensity of HPV $+/$ Cyt women for the same primary screening frequency and switch-age provided only minor improvements in cancer risk reductions (i.e., $\leqslant 1 \%)$. We found similar patterns when we evaluated the reduction in cervical cancer incidence compared to the current Norwegian cytology-based guidelines, ranging from $26.0 \%(21.6-31.9 \%)$ to $69.7 \%(67.5-72.8 \%)$ (Supplementary Figure 8). The projected number of women who developed cervical cancer per 1000 women screened over a lifetime was $3.75(2.46-4.52)$ women for the current Norwegian guidelines, and ranged from $1.19(0.69-1.49)$ to $2.82(1.75-3.50)$ women for the alternative HPV-based strategies (Supplementary Figure 9).

Resource use (i.e., the total number of cytology and HPV tests, the number of precancer treatments, and the number of colposcopy referrals, per 1000 women screened over a lifetime) varied considerably across the strategies (Supplementary Figures 10-12). As expected, the total number of cytology and HPV tests was most influenced by the primary screening frequency (Supplementary Figure 10). In contrast, the number of colposcopy referrals and precancer treatments per 1000 women screened over a lifetime was most influenced by the follow-up

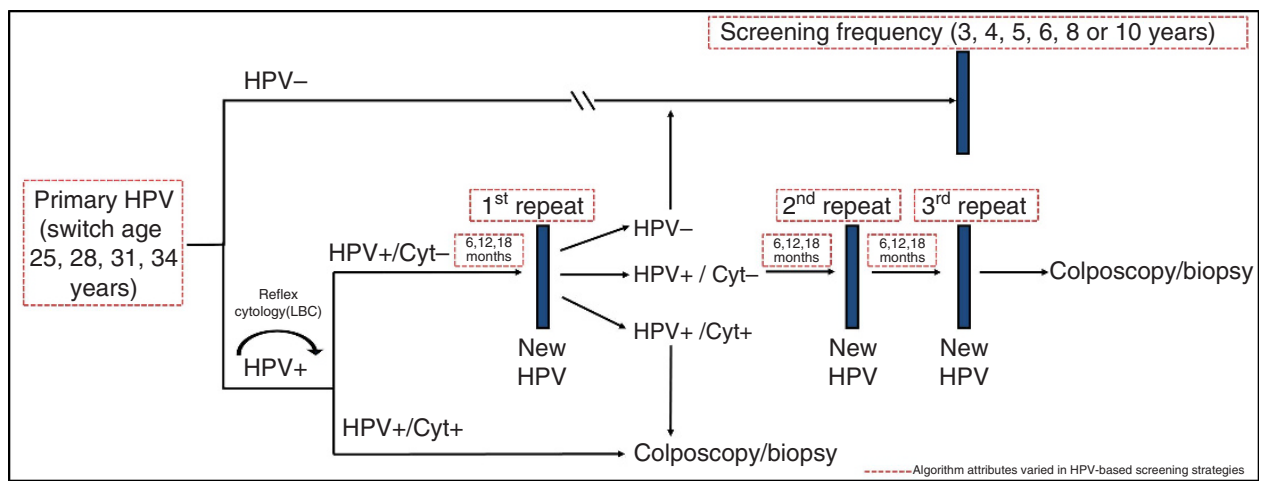

Figure 1. Flow diagram for alternative primary HPV-based screening algorithms. Dashed red boxes indicate cervical cancer screening strategies that were varied in the analysis. Women who are HPV + /Cyt - receive a new HPV test in 6, 12 or 18 months, with a reflex cytology only for those women who are HPV-positive. Cyt = cytology; HPV = human papillomavirus; HPV + /Cyt $-=$ HPV-positive and cytology-negative; HPV +/Cyt $+=$ HPVpositive and cytology result indicating atypical squamous cells of undetermined significance or more severe; LBC $=$ liquid-based cytology. 
Reduction in cancer incidence (compared to no screening)

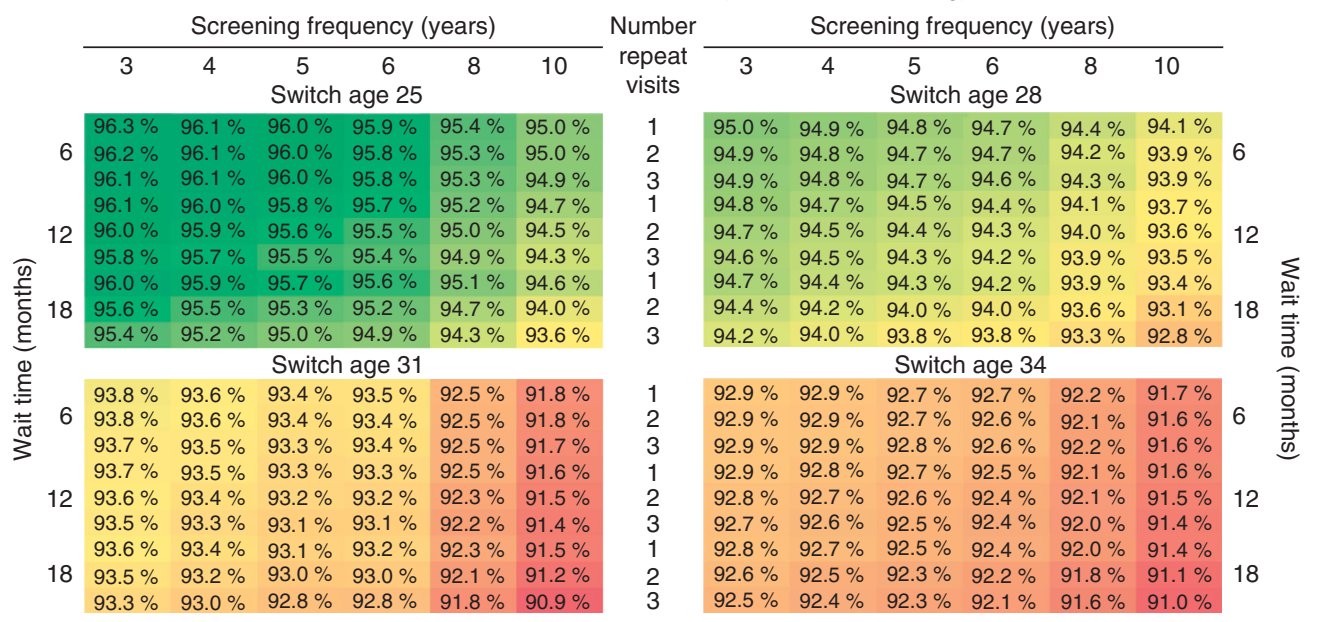

Figure 2. Reduction (\%) in lifetime cervical cancer incidence compared to no screening for the alternative HPV-based strategy algorithms. Variations in the HPV-based strategy algorithm differed by the primary screening frequency $(3,4,5,6,8$ or 10 years), the alternative ages that women switch from cytology- to HPV-based screening (age 25, 28, 31 or 34 years), the number of persistent HPV-positive, cytology-negative results required to prompt referral to colposcopy (1, 2 or 3 persistent HPV $+/$ Cyt - result(s)) and whether the strategy should require women to wait 6, 12 or 18 months between the repeat testing. The current cytology-based approach in Norway is projected to reduce lifetime cancer risk by $87.7 \%$ (uncertainty bounds: $86.6-88.7 \%$ ) compared to no screening. Heat map formatting indicates low (dark red) to high values (dark green).



Figure 3. Relative colposcopy referral rates compared to current triennial cytology-based algorithm in Norway for the alternative HPV-based strategy algorithms. Variations in the HPV-based strategy algorithm differed by the primary screening frequency $(3,4,5,6,8$ or 10 years), the alternative ages that women switch from cytology- to HPV-based screening (age 25, 28, 31 or 34 years), the number of persistent HPV-positive, cytology-negative results required to prompt referral to colposcopy $(1,2$ or 3 persistent HPV + /Cyt - result(s)) and whether the strategy should require women to wait 6, 12 or 18 months between the repeat testing. The current cytology-based approach in Norway is projected to require 567 (uncertainty bounds: 483-617) colposcopy referrals per 1000 women over their lifetime. Heat map formatting indicates low (dark green) to high values (dark red).

management approach for HPV + /Cyt - women (Supplementary Figures 11 and 12). Importantly, all HPV-based strategies we considered were associated with increases in colposcopy referral rates (i.e., up to $331 \%(312-351 \%)$ ) compared to the level projected by the current Norwegian cytology-based guidelines (i.e., 567 (483617) per 1000 women screened over a lifetime) (Figure 3 and Supplementary Figures 4 and 5). In general, switching at an earlier age to HPV-based screening or implementing a shorter primary screening frequency increased colposcopy referral rates; however, referral rates could be tempered (or exacerbated) when coupled with less (or more) intensive follow-up management approaches for $\mathrm{HPV}+/ \mathrm{Cyt}-$ women. For example, for an HPV-based strategy involving 5-yearly screening starting at age 34 years, we projected that colposcopy referral rates could increase by $145 \%$ (135-158\%) assuming the most intensive follow-up approach, but only increase by $31 \%(25-38 \%)$ for the least intensive follow-up approach. Reducing the number of repeat visits to confirm HPV persistence among HPV +/Cyt- women from 3 to 1 offset the increases in colposcopy referral rates if coupled with increasing the wait time between repeat testing (i.e., from 6 to 18 months).

The number of colposcopy referrals to prevent one additional cervical cancer (compared to no screening) increased as the intensity of the HPV-based screening strategy increased, ranging from $21(18-33)$ to $82(67-122)$ colposcopies per averted cervical cancer case (Figure 4 and Supplementary Figures 6 and 7). To maintain the current colposcopy referrals per averted cancer case projected by the Norwegian cytology-based strategy (i.e., 21 (18-31) colposcopies per averted cancer case) generally involved selecting an HPV-based strategy with the least intensive $\mathrm{HPV}+/ \mathrm{Cyt}-$ management approach or lengthening the primary 




Figure 4. The number of colposcopy referrals per cervical cancer case prevented for the alternative HPV-based strategy algorithms. Variations in the HPV-based strategy algorithm differed by the primary screening frequency $(3,4,5,6,8$ or 10 years), the alternative ages that women switch from cytology- to HPV-based screening (age 25, 28, 31 or 34 years), the number of persistent HPV-positive, cytology-negative results required to prompt referral to colposcopy (1,2 or 3 persistent HPV + /Cyt - result(s)) and whether the strategy should require women to wait 6, 12 or 18 months between the repeat testing. The current cytology-based approach in Norway is projected to require 20.8 (uncertainty bounds: 18.1-31.0) colposcopy referrals per cancer prevented. Heat map formatting indicates low (dark green) to high values (dark red).

screening frequency (Figure 4; green shaded region). The additional number of colposcopies required to prevent one additional cervical cancer compared to the current Norwegian guidelines was substantially higher, ranging from 38 (11-76) to 735 (637-969) (Supplementary Figure 13).

Uncertainty analysis. The impact of alternative HPV-based strategy algorithms on the reductions in lifetime cervical cancer risk was greatly influenced by the HPV DNA test sensitivity (Supplementary Figures 14 and 15). When we reduced the clinical HPV test sensitivity to $86 \%(81-89 \%)$, reductions in lifetime risk decreased, ranging from $84.2 \%(82.6-85.2)$ to $94.6 \%(94.1-95.2 \%)$ compared to no screening (Supplementary Figure 14). Importantly, under this scenario the primary screening frequency rather than HPV switching age, contributed to the largest impacts on cancer risk reductions. For example, lengthening the primary screening frequency from 3 to 10 years was associated with a 5-8\% lower absolute reduction in cancer risk, while delaying HPV-based screening initiation from age 25 to 34 years resulted in $<4 \%$ absolute difference in the risk reductions.

We found that relative colposcopy referrals rates were sensitive to the baseline comparator strategy, while reducing HPV test sensitivity or assuming perfect colposcopy performance had minimal impacts on colposcopy referral rates (Supplementary Figures 16-18). For example, when we compared colposcopy referrals rates with the current triennial cytology-based guidelines recommended in the US, we found that approximately two-thirds of the HPV-based strategy algorithms we considered had higher colposcopy referrals (Supplementary Figure 18). Finally, reducing HPV test sensitivity resulted in a lower ratio of the number of colposcopy referrals per cancer prevented across algorithms (ranging from 18 (15-26) to 69 (58-102); Supplementary Figure 19), while assuming perfect colposcopy characteristics yielded a higher ratio (ranging from 22 (18-37) to 87 (73-135); Supplementary Figure 20).

\section{DISCUSSION}

Modifying a well-established screening programme requires the evaluation of not only the expected benefits, but also the impact on constrained resources and potential harms to women. In this analysis, we use a model-based approach to project the magnitude of health benefits and colposcopy utilisation associated with varying levers associated with primary HPV-based screening (i.e. primary screening frequency, the age to switch to primary HPV testing, and the follow-up intensity for HPV + /Cyt - women). We found that while HPV-based screening uniformly decreased cancer incidence, these improvements generally required increases in colposcopy referral rates. However, our analysis identified the most important levers to help maintain or improve the expected cancer benefits compared to the current screening program while controlling colposcopy referral rates. Such strategies generally involved increasing the number of repeat tests and/or increasing the length of time between repeat testing prior to prompting colposcopy referral.

Worldwide, commonly proposed HPV-based algorithms involve switching to HPV testing at age $>30$ years every 5 years with reflex cytology for HPV-positive women, with a single repeat HPV-test at 12 months for cytology-negative women (Nygard et al, 2013; Ronco et al, 2014). We found that, compared to current cytology-based approaches, this algorithm is expected to reduce cancer incidence, but will likely increase colposcopy referral rates (Figure 3 and Supplementary Figure 18). Arguments for delaying switching to HPV-based screening to after age 30 years generally stems from the higher proportion of transient HPV infections at younger ages and concerns about unnecessary screening procedures. A study from the Netherlands (de Kok et al, 2014) evaluated the potential cancer preventive benefit that could be achieved by switching to primary HPV testing at age 30 rather than age 35 years. This study found that a maximum of $28 \%$ of cervical cancer cases in women aged 30-35 years could have been prevented; however, the study did not evaluate the resource requirements to achieve these benefits and emphasised the need to balance this potential benefit with potential harms stemming from unnecessary screening procedures. Our study explicitly quantifies these trade-offs and demonstrates that excess colposcopy referrals can be mediated through less intensive follow-up algorithms for $\mathrm{HPV}+/ \mathrm{Cyt}$ - women, even when initiating primary HPV testing at age 25 years. The number of repeat visits and length of time between visits serve as algorithmic levers to offset increases in colposcopy referrals by allowing time for HPV infections to clear while actively monitoring women to protect against interval 
progression. In addition, eliminating primary cytology-based screening by initiating screening at age 25 years with primary HPV testing allows policy makers to unify and simplify a screening programme while simultaneously improving cancer prevention. Ultimately, decision makers have to decide what constitutes an acceptable balance between benefits and harms of screening.

Comparison with other studies. To our knowledge, this is the first study to evaluate the long-term health and colposcopy use trade-offs associated with a multitude of candidate primary HPV and triage testing strategies. Randomised controlled trials comparing a single HPV testing strategy with or without a cytology co-test in primary screening have been conducted, finding that HPVbased screening allows longer protection against development of precancer and cancer. A recent 14-year follow-up analysis of the Dutch randomised trial (Dijkstra et al, 2016) found that the primary screening frequency may be safely extending beyond five years using primary HPV-based screening. This study also found that the 14-year risk of developing CIN3 + for screen-positive women differed, and was much higher among HPV-positive women compared to HPV-negative women at baseline, suggesting that active management of all HPV-positive women is required. Three of the European trials found no increases in colposcopy referral rates associated with primary HPV testing (Ronco et al, 2014), while other trials have reported increases in colposcopy rates, especially among younger women (i.e., $<35$ years) (Leinonen et al, 2009; Coldman et al, 2015). Two Dutch analyses that evaluated up to 14 alternative management approaches for screen-positive women (Rijkaart et al, 2012; Dijkstra et al, 2014) found that select triage strategies may control colposcopy rates (improving the positive predictive value), with only minor losses in the negative predictive values of high-grade lesions. Another Dutch study found that primary HPV screening was preferred over cytology-based screening; however, the most efficient strategies often involved using only cytology to triage HPV + /Cyt - women as the number of false positives associated with HPV triage outweighed the benefits (van Rosmalen et al, 2012). Although a cytology-only triage approach would likely be an alternative strategy to control colposcopy referrals, this was not currently on the policy table in Norway. These empirical studies, while important and in line with our findings, report on health and resource consequences that do not reflect repeated rounds of screening with primary HPV-testing over a lifetime, and differ considerably in control-arm strategy, primary screening frequency, and follow-up management of screen-positive women.

Disease simulation models provide a unique opportunity to quantify the long-term health benefits and economic consequences associated with candidate screening algorithms, by simulating a hypothetical cohort of women over their lifetime. Simulation modelling has been readily used to assess the value of implementing primary HPV testing (Mendes et al, 2015); however, the majority of these studies capture economic outcomes using the single composite outcome of monetary costs, rather than reporting resource use in natural units such as colposcopy referrals. A recent Australian study (Smith et al, 2016) projected the impact of transitioning from the current biennial cytology-based programme to a single proposed 5-yearly HPV-based strategy on resource use, finding that colposcopy referrals are expected to increase for the first three rounds of HPV-based screening for women not vaccinated against HPV infections. For countries that recommend a lower threshold to prompt colposcopy referral (e.g., LSIL directly to colposcopy), or a more frequent primary cytology screening frequency (such as Australia), switching to primary HPV testing will likely have a smaller impact on comparative resource use. In general, and similar to our findings, studies that have quantified resource use in natural units have found that the differential impact of candidate strategy algorithms and testing approaches on health benefits are small, while resource use may vary considerably (Kim et al, 2002; Stout et al, 2008; Pedersen et al, 2016), highlighting the value of using simulation modelling to help inform the trade-offs of alternative screening algorithms.

Limitations. Several limitations are worth mentioning. We did not evaluate the health benefit and resource use trade-offs among HPV vaccinated cohorts. In Norway, the first vaccinated cohorts will not reach screening age until 2022; therefore, identifying HPVbased strategies that control colposcopy resource use among unvaccinated cohorts is the greatest priority; a comprehensive analysis evaluating optimal screening algorithms among vaccinated cohorts will be the focus of future analyses. In addition, we restricted our analysis to long-term, non-monetary outcomes. Cost-effectiveness analyses that evaluate the cost per qualityadjusted life year gained are necessary to provide a more comprehensive identification of the optimal algorithm for a given setting. These studies will be required to ensure that the reduction in colposcopy referrals achieved by choosing a less intensive followup approach for HPV + /Cyt - women are not outweighed by utility decrements that may stem from a potential increase in a woman's anxiety while undergoing diagnostic work-up, or from the financial costs associated with increasing the number of repeat follow-up visits (especially when considering women's time and travel costs).

Due to the absence of data informing future screening behaviour associated with alternative algorithms, we did not consider the role of loss-to-follow-up after an abnormal result, which may increase as an algorithm requires additional persistent results. For decisionmakers concerned with a potential increase in loss-to-follow-up due to requiring multiple repeat visits or increasing the wait time prior to diagnostic confirmation, our results show that policy makers can select the appropriate setting-specific levers to triage HPV-positive women in order to control colposcopy referral rates, without losing health benefits.

Although we also did not evaluate all possible triage approaches for HPV-positive women, such as HPV genotyping (Stoler et al, 2015a), similar approaches, such as less intensive follow-up management of screen-positive women, can be employed to control colposcopy referrals. As expected, we found that relative colposcopy use is dependent on the baseline comparator strategy; therefore, the exact magnitude of relative increases may not be generalisable outside settings that recommend guidelines comparable to the Norwegian approach. For countries with similar algorithms to the United States that involve referring women with LSIL results directly to colposcopy, we found that switching to primary HPV-based screening would not necessarily require current systems to scale-up colposcopy resources to accommodate new screening algorithms, particularly for certain screening frequencies, primary HPV switching ages and HPV + /Cyt management combinations (Supplementary Figure 18).

Policy implications. The health and resource trade-offs identified in this study may aid decision makers in designing the optimal HPV-based screening algorithm given local capacity constraints and preferences for balancing the benefits and harms of screening. In the short-term, resource constraints may limit the choice among potential strategies, while in the long-term, scaling up programmes to accommodate algorithm requirements may be achievable. Our analysis highlights the benefit of switching to primary HPV testing at an earlier age, which can be implemented without considerable increases in colposcopy referral rates if paired with less intensive follow-up approaches for HPV + /Cyt - women. In deciding the optimal follow-up of HPV + /Cyt - women, decision-makers should also consider potential harms associated with less intensive approaches. For example, women who are HPV +/Cyt - may experience anxiety by having to wait $12-18$ months for repeat testing (once or twice), rather than immediate adjudication. In a British study (Waller et al, 2007) that conducted 
in-depth interviews with 30 women who tested HPV $+/$ Cyt - at their primary screen and who attended repeat HPV testing in 12 months, most women did not experience considerable anxiety while waiting for the first repeat HPV test, yet preferred immediate resolution with colposcopy referral rather than a second repeat HPV test. Moreover, women showed a greater emotional impact of a repeat HPV-positive result; as such, it may be better to increase the wait-time prior to repeat testing rather than the number of persistent $\mathrm{HPV}$-positive results required to prompt a colposcopy referral.

\section{CONCLUSIONS}

Strategies involving primary HPV-based screening for unvaccinated women are expected to improve health benefits with respect to cancer reduction, but may also increase colposcopy utilisation. Our analysis highlights ways in which HPV testing can simultaneously yield large improvements in population health benefits while controlling colposcopy referral rates.

\section{ACKNOWLEDGEMENTS}

This work was supported in part by The Norwegian Research Council (grant number 238042 (EAB and KP)); and by the U.S. National Cancer Institute (R01CA160744 (PI: JJK)). The funders had no role in the conduct of this research.

\section{CONFLICT OF INTEREST}

The authors declare no conflict of interest.

\section{REFERENCES}

Arbyn M, Bergeron C, Klinkhamer P, Martin-Hirsch P, Siebers AG, Bulten J (2008) Liquid compared with conventional cervical cytology: a systematic review and meta-analysis. Obstet Gynecol 111: 167-177.

Burger EA, Ortendahl JD, Sy S, Kristiansen IS, Kim JJ (2012) Costeffectiveness of cervical cancer screening with primary human papillomavirus testing in Norway. Br J Cancer 106: 1571-1578.

Burger EA, Sy S, Nygard M, Kim JJ (2016) The cost-effectiveness of cervical self-sampling to improve routine cervical cancer screening: The importance of respondent screening history and compliance. Cancer Epidemiol Biomarkers Prev 26(1): 95-103.

Campos NG, Burger EA, Sy S, Sharma M, Schiffman M, Rodriguez AC, Hildesheim A, Herrero R, Kim JJ (2014) An updated natural history model of cervical cancer: derivation of model parameters. Am J Epidemiol 180: 545-555.

Cancer Registry of Norway (2011) Cancer in Norway 2009. Special Issue: Cancer screening in Norway. Oslo, Norway.

Coldman AJ, Phillips N, van Niekerk D, Smith L, Krajden M, Cook D, Quinlan DJ, Ehlen T, Miller D, Stuart GC, Peacock S, Elwood Martin R, Franco EL, Ogilvie G (2015) Projected Impact of HPV and LBC Primary Testing on Rates of Referral for Colposcopy in a Canadian Cervical Cancer Screening Program. J Obstet Gynaecol Can 37: 412-420.

Cuzick J, Mayrand MH, Ronco G, Snijders P, Wardle J (2006) Chapter 10: New dimensions in cervical cancer screening. Vaccine 24(Suppl 3): S3/90-97.

de Kok IM, van Rosmalen J, Rozemeijer K, Penning C, van Ballegooijen M (2014) How many cervical cancer cases can potentially be prevented using a more sensitive screening test at young age? Int J Cancer 134: 460-466.

Dijkstra MG, van Niekerk D, Rijkaart DC, van Kemenade FJ, Heideman DA, Snijders PJ, Meijer CJ, Berkhof J (2014) Primary hrHPV DNA testing in cervical cancer screening: how to manage screen-positive women? A POBASCAM trial substudy. Cancer Epidemiol Biomarkers Prev 23: 55-63.

Dijkstra MG, van Zummeren M, Rozendaal L, van Kemenade FJ, Helmerhorst TJ, Snijders PJ, Meijer CJ, Berkhof J (2016) Safety of extending screening intervals beyond five years in cervical screening programmes with testing for high risk human papillomavirus: 14 year follow-up of population based randomised cohort in the Netherlands. BMJ 355: i4924.

Jin G, LanLan Z, Li C, Dan Z (2014) Pregnancy outcome following loop electrosurgical excision procedure (LEEP) a systematic review and metaanalysis. Arch Gynecol Obstet 289: 85-99.

Kim JJ, Kuntz KM, Stout NK, Mahmud S, Villa LL, Franco EL, Goldie SJ (2007) Multiparameter calibration of a natural history model of cervical cancer. Am J Epidemiol 166: 137-150.

Kim JJ, Wright TC, Goldie SJ (2002) Cost-effectiveness of alternative triage strategies for atypical squamous cells of undetermined significance. JAMA 287: 2382-2390.

Leinonen M, Nieminen P, Kotaniemi-Talonen L, Malila N, Tarkkanen J, Laurila P, Anttila A (2009) Age-specific evaluation of primary human papillomavirus screening vs conventional cytology in a randomised setting. J Natl Cancer Inst 101: 1612-1623.

Mayrand MH, Duarte-Franco E, Rodrigues I, Walter SD, Hanley J, Ferenczy A, Ratnam S, Coutlee F, Franco EL (2007) Human papillomavirus DNA versus Papanicolaou screening tests for cervical cancer. N Engl J Med 357: 1579-1588.

Mendes D, Bains I, Vanni T, Jit M (2015) Systematic review of model-based cervical screening evaluations. BMC Cancer 15: 334.

Moyer VA (2012) Screening for cervical cancer: U.S. Preventive Services Task Force recommendation statement. Ann Intern Med 156: 880-891w312.

Naber SK, de Kok IM, Matthijsse SM, van Ballegooijen M (2016) The potential harms of primary human papillomavirus screening in overscreened women: a microsimulation study. Cancer Causes Control 27: 569-581.

Nanda K, McCrory DC, Myers ER, Bastian LA, Hasselblad V, Hickey JD, Matchar DB (2000) Accuracy of the Papanicolaou test in screening for and follow-up of cervical cytologic abnormalities: a systematic review. Ann Intern Med 132: 810-819.

Norwegian Technical Appendix (2017) Harvard Cervical Cancer Natural History Model Calibration and Costing Approach for Norway. Available at: http://www.med.uio.no/helsam/english/research/projects/preventivestrategies-hpv/17-harvardmodel-norway-technicalappendix.pdf (accessed on 10 May 2017).

Nygard M, Andreassen T, Berland J, Hagen B, Hagmar B, Iversen O-E, Juvkam K-H, Kristiansen IS, Lønnberg SV, Sørby SW, Vintermyr OK, Aarseth H-P (2013) HPV testing in primary screening for cervical cancer. Controlled implementation and evaluation of improved health service. The Norwegian Directorate of Health: Oslo, Norway.

Pedersen K, Burger EA, Sy S, Kristiansen IS, Kim JJ (2016) Cost-effective management of women with minor cervical lesions: Revisiting the application of HPV DNA testing. Gynecol Oncol 143: 326-333.

Rijkaart DC, Berkhof J, van Kemenade FJ, Coupe VM, Hesselink AT, Rozendaal L, Heideman DA, Verheijen RH, Bulk S, Verweij WM, Snijders PJ, Meijer CJ (2012) Evaluation of 14 triage strategies for HPV DNA-positive women in population-based cervical screening. Int J Cancer 130: $602-610$

Ronco G, Dillner J, Elfstrom KM, Tunesi S, Snijders PJ, Arbyn M, Kitchener H, Segnan N, Gilham C, Giorgi-Rossi P, Berkhof J, Peto J, Meijer CJ (2014) Efficacy of HPV-based screening for prevention of invasive cervical cancer: follow-up of four European randomised controlled trials. Lancet 383: 524-532.

Smith MA, Gertig D, Hall M, Simms K, Lew JB, Malloy M, Saville M, Canfell K (2016) Transitioning from cytology-based screening to HPVbased screening at longer intervals: implications for resource use. $B M C$ Health Serv Res 16: 147.

Statistics Norway (2015) Available at: http://ssb.no/befolkning/statistikker/ dode/aar/2015-03-26?fane=tabell\&sort=nummer\&tabell=220943 (accessed 16 October 2015).

Stoler MH, Austin RM, Zhao C (2015a) Point-counterpoint: cervical cancer screening should be done by primary human papillomavirus testing with genotyping and reflex cytology for women over the age of 25 years. J Clin Microbiol 53: 2798-2804. 
Stoler MH, Ronnett BM, Joste NE, Hunt WC, Cuzick J, Wheeler CM (2015b) The interpretive variability of cervical biopsies and its relationship to HPV status. Am J Surg Pathol 39: 729-736.

Stout NK, Goldhaber-Fiebert JD, Ortendahl JD, Goldie SJ (2008) Trade-offs in cervical cancer prevention: balancing benefits and risks. Arch Intern Med 168: 1881-1889.

van Rosmalen J, de Kok IM, van Ballegooijen M (2012) Cost-effectiveness of cervical cancer screening: cytology versus human papillomavirus DNA testing. BJOG 119: 699-709.
Waller J, McCaffery K, Kitchener H, Nazroo J, Wardle J (2007) Women's experiences of repeated HPV testing in the context of cervical cancer screening: a qualitative study. Psychooncology 16: 196-204.

This work is published under the standard license to publish agreement. After 12 months the work will become freely available and the license terms will switch to a Creative Commons AttributionNonCommercial-Share Alike 4.0 Unported License.

Supplementary Information accompanies this paper on British Journal of Cancer website (http://www.nature.com/bjc) 